\title{
Novel combination of paraspinal keyhole surgery with a tubular retractor system leads to significant improvements in lumbar intraspinal extramedullary schwannomas
}

\author{
YUANDONG ZHUANG ${ }^{*}$, GANGFENG CAI* , CHAOFENG FU, WEIQIANG ZHANG, \\ WEI ZHAO, RUI WANG, CHUNHUA WANG, SONGSHENG SHI and CHUNMEI CHEN \\ Department of Neurosurgery, Union Hospital of Fujian Medical University, \\ Fujian Institute of Neurosurgery, Fuzhou, Fujian 350001, P.R. China
}

Received May 11, 2016; Accepted June 2, 2017

DOI: $10.3892 / \mathrm{ol} .2017 .7203$

\begin{abstract}
The aim of the present study was to investigate the efficacy of combining paraspinal keyhole surgery with a tubular retractor system for the microsurgical removal of lumbar intraspinal extramedullary schwannomas. A retrospective analysis was conducted of 56 patients with lumbar intraspinal extramedullary schwannomas who were treated using the microsurgical paraspinal keyhole approach with a tubular retractor system. The mean \pm standard deviation was calculated for the following parameters: Surgery time (96.21 $\pm 14.64 \mathrm{~min})$, hemorrhagic volume $(28.54 \pm 9.72 \mathrm{ml})$, bed rest $(2.55 \pm 0.5$ days) and hospital stay (5.68 \pm 0.72 days). Two patients presented with cerebrospinal fluid leakage and one patient exhibited a nerve root injury. At a 6-month follow-up visit, postoperative Japanese Orthopedic Association (JOA) and visual analog scale (VAS) scores were evaluated. The mean \pm standard deviation JOA scores were $12.00 \pm 2.07$ for preoperative, $14.73 \pm 2.05$ for 1 week postoperative, $20.07 \pm 2.32$ for 3 months postoperative and $21.75 \pm 2.18$ for 6 months postoperative. The improvement rate was 16.07, 47.48 and $59.77 \%$, respectively. The mean \pm standard deviation VAS scores were $6.64 \pm 1.31$ for preoperative, $3.82 \pm 1.51$ for 1 week postoperative, $2.11 \pm 1.17$ for 3 months postoperative and $1.50 \pm 1.51$ for 6 months postoperative. The JOA and VAS scores improved significantly $(\mathrm{P}<0.05)$. Magnetic resonance imaging and computed tomography were performed preoperatively, immediately following surgery and at the 6-month postoperative visit to confirm the efficacy of the resections
\end{abstract}

Correspondence to: Dr Chunmei Chen, Department of Neurosurgery, Union Hospital of Fujian Medical University, Fujian Institute of Neurosurgery, Fuzhou, Fujian 350001, P.R. China

E-mail: cmchen2009@hotmail.com

*Contributed equally

Key words: schwannoma, microsurgery, lumbar spine, paraspinal, tubular retractor system, keyhole surgery and evaluate spinal stability. No residual tumors were identified at follow-up. No alterations in the stability of the spine were observed postoperatively. The combination of the microsurgical paraspinal keyhole approach with the tubular retractor system was successful in treating lumbar intraspinal extramedullary schwannomas. The surgical approach was associated with decreased hemorrhages, decreased duration of hospital stay, faster recovery and improved postoperative maintenance of spinal stability.

\section{Introduction}

Schwannomas are common intraspinal tumors frequently observed in the cervical and lumbar regions (1). Spinal schwannomas are slow-growing lesions that primarily arise from the dorsal sensory roots, leading to changes in radicular sensory processes that may result in debilitating effects. Spinal schwannomas are commonly confined to one side of the spine. Patients commonly present with pain, with weakness being a less common symptom.

Surgery is currently the primary treatment for schwannomas and aims to restore neurological function, ambulation and bladder control, and improve life quality, with two major surgical approaches used: The posterior midline approach or the unilateral hemilaminectomy approach (2-9).

The posterior midline approach requires resecting the muscles, ligaments, spinous processes and part of the lamina. Clinical biomechanical studies have demonstrated that the posterior midline approach may cause postoperative spinal instability and long-lasting back pain, and limit the range of motion due to ligament and joint damage. In contrast, unilateral hemilaminectomy, which decreases the damage to the interspinous and supraspinous ligaments and lamina, may be associated with a decrease in the severity of these postoperative complications $(10,11)$. However, surgical vision is restricted within the unilateral spinal canal and there is limited operative space $(7,10)$. Currently, the paraspinal tubular retractor system has been commonly used in spine discectomies. It is minimally invasive to the paraspinal muscles and the vertebrae, while preserving the facet joint. Unlike unilateral hemilaminectomy, the paraspinal tubular 
retractor system offers a wide surgical area as the tubular retractor is able to adjust its angle, and provides improved access for pathological excision, which may be achieved using non-expandable retractors. In previous studies, lumbar disc herniations have been treated using the tubular retractor system (11-14). The present study introduces a novel surgical approach for lumbar intraspinal schwannomas which was observed to exhibit good clinical efficacy.

\section{Materials and methods}

Inclusion and exclusion criteria. The study described in the present study has been carried out in accordance with the Code of Ethics of The World Medical Association (the Declaration of Helsinki) for experiments involving humans. The present study was approved by, and carried out according to the Fujian Medical University (Fuzhou, China) Human Investigations or Ethics Committee protocol. Informed written consent was obtained for experimentation with all patients. The privacy rights of the patients were always observed. Two types of tumor were included in the study. Extramedullary spinal tumors with the majority of their mass residing unilaterally intraspinal were confirmed through clinical manifestations and imaging studies. Small-sized tumors were identified with a threshold transverse diameter $\leq 2 \mathrm{~cm}$ and a height that was less than or equal to the height of one vertebra in radial length. A clinical examination was conducted on all patients who were identified as potentially possessing schwannomas following review of imaging reports, in order to confirm the affected spaces.

On the basis of imaging studies, several types of schwannoma were excluded from the present study. The patients that required internal fixation following surgical removal were excluded from the present study.

Patients. A total of 56 patients (24 male and 32 female) who were admitted to the Affiliated Union Hospital of Fujian Medical University (Fuzhou, China) between November 2011 and April 2015 qualified for the present study. Patients were undergoing a microsurgical procedure using a paraspinal keyhole approach with a tubular retractor system. Pathologies of schwannomas were retrospectively analyzed. The age of disease onset ranged from 16 to 72 years, with a mean age of 45.6 years. Patients aged between 40 and 50 comprised the largest group of patients ( $42.86 \%$ or 24 cases). Two patients were selected for in depth analysis.

Clinical manifestations. Patients reported a mean disease history of 13.66 months (ranging from 1 to 31 months). Clinical manifestations varied with location and size of the tumors as well as whether spinal cord compression was evident $(15,16)$. Early symptoms included irritation, itchiness, palsy and sense of expansion. Symptoms associated with cord compression were prominent in the late phase. Specific distributions of the patients' symptoms were summarized as follows: 32 cases of radiculalgia, 34 of paresthesia, 17 of dyskinesia and 4 bowel or bladder dysfunction cases.

Imaging examinations. All the patients received plain and T1-weighted magnetic resonance imaging (MRI) and computed tomography (CT). MRI clearly revealed the shape of tumors and compression-induced shifting of the spine (17-20). CT scans clearly revealed signs of foramen expansion, bone destruction and tumor outgrowth (21). MRI, T1-weighted MRI and CT three-dimensional reconstructions were conducted postoperatively. T1-weighted MRI and CT scans were used to assess the condition of tumor resections and the effects of surgery on tumor-induced cord compressions. CT three-dimensional reconstructions were used to reveal the effect of surgery on the bony structure of the vertebrae.

Surgical procedure. Patients received general anesthesia via endotracheal intubation, while in a prone position and with a vacant abdomen (on a high-intensity foam pad). Following a detailed analysis of the tumor location and size based on the preoperative MRI and CT, the intervertebral space was confirmed. The surgical approach that would cause the least damage to the paraspinal muscles and nerve roots was selected following assessment of the impact of the approach on spinal stability. The location of the corresponding intervertebral space was marked at the midline using a paperclip, which indicated the site of incision when X-rayed. Following preoperative preparation, an incision $(1.5-2.0 \mathrm{~cm})$ was made parallel to the spine at $2.0 \mathrm{~cm}$ to the midline of the spinous process. The incision site may have varied according to the location, size and the specific position detected by X-rays of the tumor. A Kirschner wire was inserted into the incision under the guidance of the C-arm X-ray machine. Blunt dissection was guided gradually to form a surgical pathway via the multifidus gap using the tubular retractor system. The surgical pathway was fixated when it reached the surface of the lamina. The superficial soft tissue of the lamina and fiavum ligament were bluntly dissected under the microscope. Part of the lamina was removed using a micro drill to reveal the spinal dura mater. Tumor resection and nerve decompression were completed under clear and full vision following adequate microscopic adjustment (Fig. 1).

Response evaluation criteria. The length of the surgery and any signs of hemorrhage were evaluated during surgery. Postoperative evaluations included the length of bed rest, the duration of the hospital stay and any postoperative complications. Follow-up visits ranged from 6 months to 47 months, with a mean duration of 25.61 months. Several parameters were measured preoperatively and at 1 week, 3 months and 6 months postsurgery. These parameters included the Japanese Orthopedic Association (JOA) score, the JOA improvement rate [JOA postoperative improvement $(\%)=($ postoperative - preoperative JOA score $) /(29$ - preoperative JOA score $)$ x 100\%] and the visual analog scale (VAS) score. MRI and CT scans were performed preoperatively, immediately following surgery and at the 6-month postoperative visit to confirm the efficacy of the resections and evaluate spinal stability.

Statistical analysis. Data are expressed as the mean \pm standard deviations. JOA and VAS scores at distinct time periods were compared using standard and repeated measures analysis of variance; multiple comparison between the groups was 


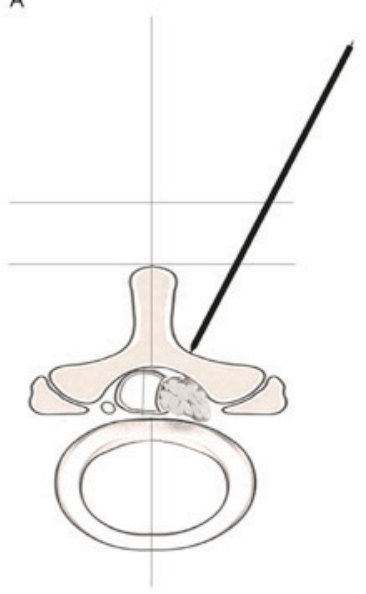

C

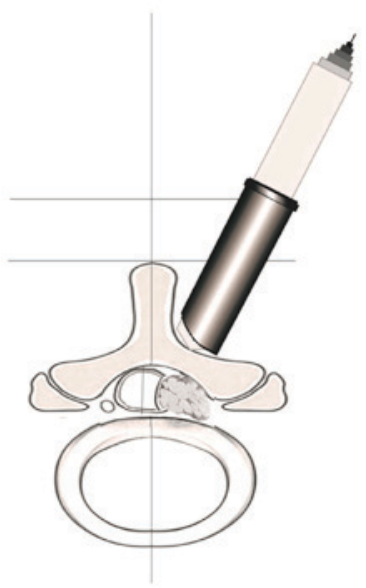

B

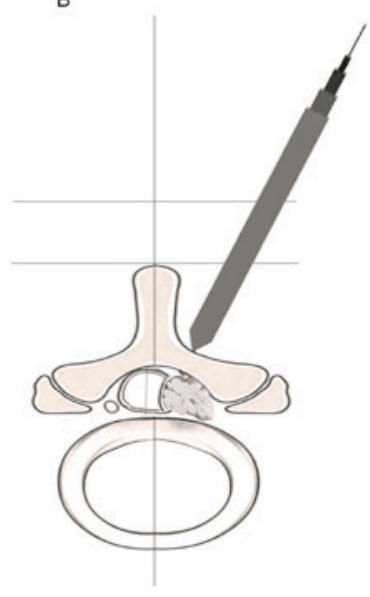

D

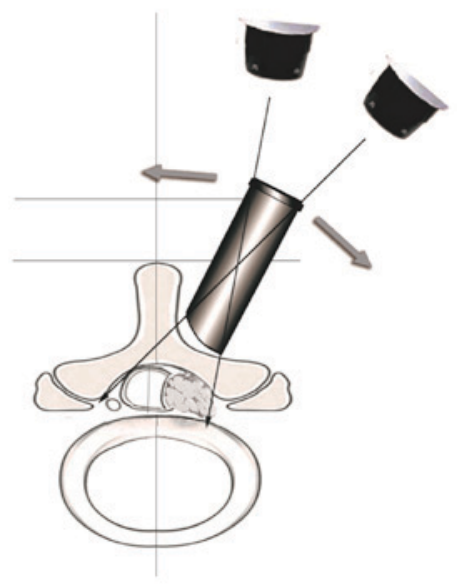

Figure 1. Schematic diagram of paraspinal keyhole surgery with a tubular retractor system. (A) Paraspinal skin was incised and a Kirschner wire was inserted into the indicated intervertebral space. (B) Sequential dilators were inserted to bluntly separate the paraspinal muscles. (C) A surgical tubular retractor was inserted, prior to the surgical pathway being fixated into the specific intervertebral space. (D) The inner retractor was withdrawn and the microscope was introduced.

performed using the S-N-K method. Analyses were performed using SPSS software (version 18.0; SPSS Inc., Chicago, IL, USA). $\mathrm{P}<0.05$ was considered to indicate a statistically significant difference.

\section{Results}

Example case reports. Two cases were selected for in-depth analysis. Case I. A 33-year-old male exhibited repeat right leg pain and numbness for 5 months becoming aggravated over the course of 5 days. The patient reported no symptoms of limb weakness, unsteady gait or bowel and bladder dysfunctions. The preliminary examination revealed a negative straight leg raising sign in the bilateral lower extremities as well as a normal pulse in the bilateral dorsalis pedis. MRI indicated a space-occupying lesion in the lumbar 4-5 transpinal canal (2.9x1.6x1.4 cm). The patient underwent microsurgery using the paraspinal keyhole approach for lumbar intraspinal extramedullary schwannomas combined with the tubular retractor system. This approach relieved the pain postoperatively (Fig. 2).
Table I. Surgery results and postoperative data.

\begin{tabular}{lccc}
\hline Op time (min) & $\begin{array}{c}\text { Intra-op } \\
\text { hemorrhage }(\mathrm{ml})\end{array}$ & $\begin{array}{c}\text { Time in } \\
\text { bed (days) }\end{array}$ & $\begin{array}{c}\text { Length of } \\
\text { hospital } \\
\text { stay (days) }\end{array}$ \\
\hline $96.21 \pm 14.64$ & $28.54 \pm 9.72$ & $2.55 \pm 0.5$ & $5.68 \pm 0.72$ \\
\hline
\end{tabular}

Values are the mean \pm standard deviation. Op, operative.

Case II. A 16-year-old male exhibited lower back pain, left leg pain and numbness for 6 months. No symptoms of limb weakness, unsteady gait or bowel and bladder dysfunctions were reported. The preliminary examination revealed a negative straight leg raising sign in bilateral lower extremities as well as a normal pulse in the bilateral dorsalis pedis. MRI indicated a space-occupying lesion in the lumbar 2 transpinal canal $(1.5 \times 1.0 \times 0.9 \mathrm{~cm})$. The patient underwent microsurgery using the paraspinal keyhole intervention for lumbar intraspinal extramedullary schwannomas combined with the tubular retractor system. Pain and numbness were alleviated postoperatively and the pain was eliminated by the 3 -month follow-up visit (Fig. 3).

Study results. The operative time ranged between 75 and $126 \mathrm{~min}$, with a mean time of $96.21 \mathrm{~min}$. Intraoperative hemorrhages ranged between 25 and $65 \mathrm{ml}$, with a mean volume of $38.54 \mathrm{ml}$. No blood transfusions were performed. Patients concluded bed rest following a mean of $2.55 \pm 0.5$ days postoperatively and ambulated with a waist guard (Table I). The complication rate was low, with only 2 patients demonstrating cerebrospinal fluid leakage and 1 patient exhibiting nerve root injury. The preoperative lumbar JOA scores were 12.00 \pm 2.07 and steadily increased at the 1-week $(14.73 \pm 2.05), 3$-month $(20.07 \pm 2.32)$ and 6 -month $(21.75 \pm 2.18)$ follow-up visits. The rate of improvement in the JOA scores also increased, with the 1-week (47.8\%) and 6-month $(57.35 \%)$ postoperative scores increasing over the preoperative (16.07\%) JOA scores (Table II and Fig. 4). The preoperative VAS score $(6.64 \pm 1.31)$ was higher than the postoperative scores and steadily declined at 1 week $(3.82 \pm 1.51), 3$ months $(2.11 \pm 1.17)$ and 6 months $(1.50 \pm 1.51)$ postsurgery. There was a significant difference between the preoperative and postoperative data $(\mathrm{P}<0.05)$. During the 6 -month follow-up, no recurrent tumors appeared in any of the 56 patients. No atrophy was observed in the spinal physiology and there were no reports of damage induced by paraspinal muscle injuries.

\section{Discussion}

Intraspinal extramedullary schwannoma is one of the most common intraspinal tumors $(1,22)$. It is classified anatomically into extramedullary, paraspinal and intradural-extramedullary schwannomas, and pathologically into Schwann cell tumors, neurofibromas and malignant schwannomas. On the basis of its association with the spinal dura mater, schwannomas are further classified as subdural-extramedullary, 
Table II. Preoperative and postoperative JOA and VAS scores.

\begin{tabular}{lcccc}
\hline Scores & Pre-op & 1 week post-op & 3 months post-op & 6 months post-op \\
\hline JOA & $12.00 \pm 2.07$ & $14.73 \pm 2.05^{\mathrm{a}}$ & $20.07 \pm 2.32^{\mathrm{a}}$ & $21.75 \pm 2.18^{\mathrm{a}}$ \\
JOA improvement $(\%)$ & & 16.07 & 47.48 & 59.77 \\
VAS & $6.64 \pm 1.31$ & $3.82 \pm 1.51^{\mathrm{b}}$ & $2.11 \pm 1.17^{\mathrm{b}}$ & $1.50 \pm 1.51^{\mathrm{b}}$ \\
\hline
\end{tabular}

${ }^{a} \mathrm{P}<0.05$ comparing JOA scores with the previous evaluation; ${ }^{\mathrm{b}} \mathrm{P}<0.05$ comparing VAS score with the previous evaluation. Values are the mean \pm standard deviation. JOA, Japanese Orthopedic Association Score; VAS, visual analog scale; op, operative.
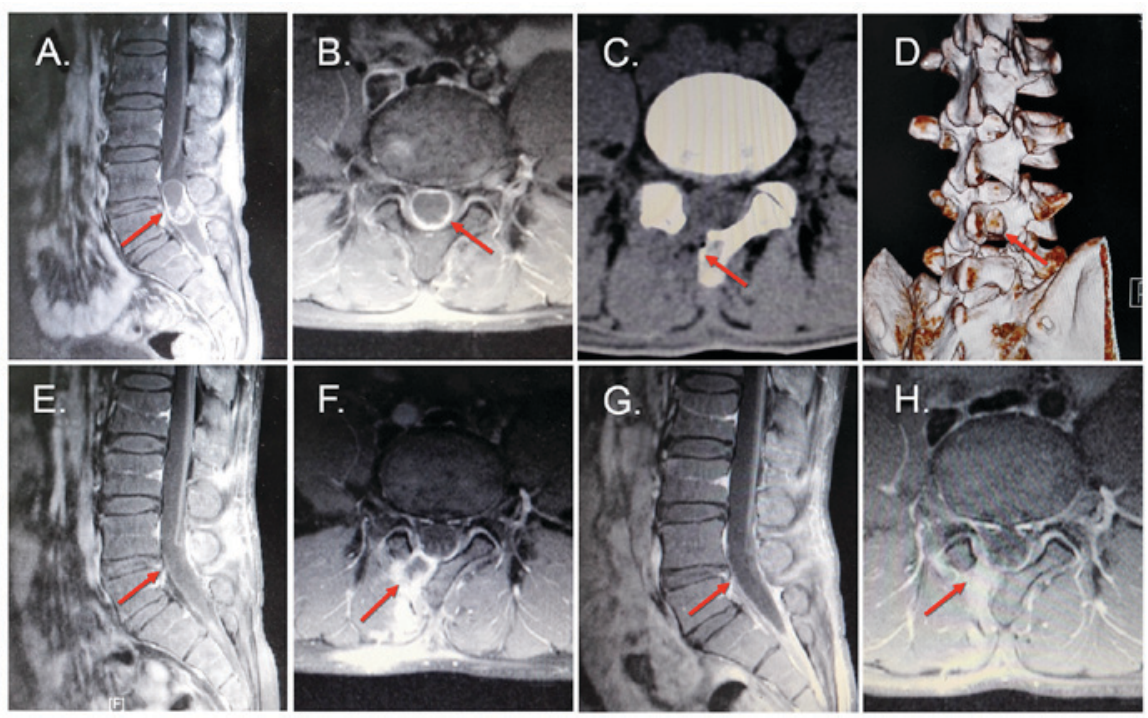

Figure 2. Images captured from case I. A 33-year-old male presented with right leg pain and numbness for 5 months and the syndrome became aggravated over 5 days. Preoperative T1-weighted (A) sagittal and (B) axial MRI images revealing a space-occupying lesion in the lumbar 4-5 transpinal canal (2.9x1.6x1.4 cm). (C) Immediate postoperative CT scan and (D) three-dimensional reconstruction demonstrating the surgical path of the tubular retractor system and paraspinal keyhole to the tumor. (E) The 3-day postoperative T1-weighted sagittal and (F) axial MRI images revealed the surgical path, demonstrating that the tumor was excised cleanly. (G) The 6-month postoperative T1-weighted sagittal and (H) axial MRI images reveal no recurrence of the tumor. The paraspinal muscle recovered well. MRI, magnetic resonance imaging; CT, computed tomography.
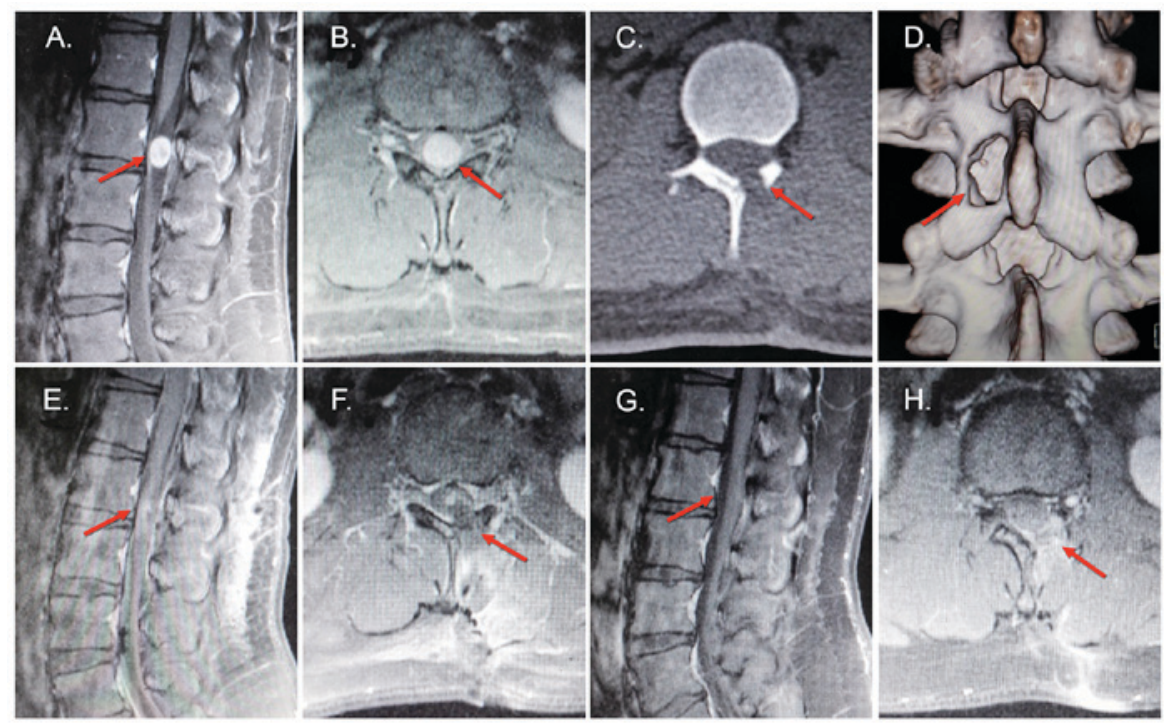

Figure 3. Images captured from case II. A 16-year-old male presented with low back pain, left leg pain and numbness for 6 months. Preoperative T1-weighted (A) sagittal and (B) axial MRI images revealing a space-occupying lesion in the lumbar 2 transpinal canal $(1.5 \times 1.0 \times 0.9 \mathrm{~cm})$. (C) Immediate postoperative CT scan and (D) three-dimensional reconstruction demonstrating the surgical path of the tubular retractor system and paraspinal keyhole to the tumor. (E) The 3-day postoperative T1-weighted sagittal and (F) axial MRI images revealed the surgical path, demonstrating that the tumor was excised cleanly. (G) The 6-month postoperative T1-weighted sagittal and (H) axial MRI images revealed no recurrence of the tumor. The paraspinal muscle recovered well. MRI, magnetic resonance imaging; CT, computed tomography. 


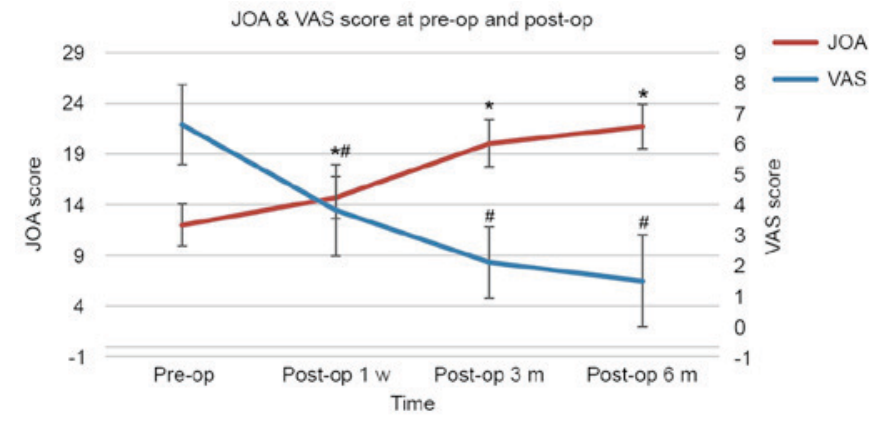

Figure 4. Preoperative and postoperative JOA and VAS scores. Over time, the VAS score decreased gradually and the JOA score increased gradually. ${ }^{*} \mathrm{P}<0.05$ when comparing JOA scores with that of previous evaluation ${ }^{\#} \mathrm{P}<0.05$ when comparing VAS score with that of previous evaluation. JOA, Japanese Orthopedic Association Score; VAS, visual analog scale; op, operative; w, week; m, month.

intradural-extramedullary, extradural, paraspinal and intramedullary (22). Intraspinal schwannoma is a slowly developing tumor with insidious onset, which lacks specific early stage signs and symptoms. The most common clinical manifestation is radiating pain due to radicular lesions caused by tumor infiltration. Unfortunately, misdiagnosis is common. The majority of schwannomas are benign tumors that are insensitive to chemotherapy, therefore prompt surgical resection following diagnosis is the optimal solution $(22,23)$.

Total posterior laminectomy combined with tumor resection is the most common surgical approach for schwannomas. This method removes the lamina, spinous process, interspinous ligament and paraspinal soft tissue, completely exposing the structures inside the spine. The approach enables surgical manipulation for various types of intraspinal extramedullary schwannoma in distinct locations $(2,3)$. However, postoperative complications may include pain, decreased stability of the spine and cerebrospinal fluid leakage (24). In certain patients, the zygapophyseal joints are excised, warranting internal fixation and fusion following tumor resection to decrease the risk of iatrogenic spinal deformity $(25,26)$. A number of previous studies have demonstrated that internal fixation and fusion increase the risk of disc degeneration of the nearby vertebrae (27-30).

Park and Heller (31) suggested that a surgical approach should cause minimum damage to the nearby nerves and blood vessels while maximally exposing the tumors and important structures surrounding them. It was reported that between 60 and $70 \%$ of the intraspinal extramedullary schwannomas were located laterally or posterolaterally, therefore removing part of the lamina was also an option (32). Hemilaminectomy preserves the interspinous and supraspinous ligaments as well as the intervertebral joints and the unilateral paraspinal muscles. In addition, this surgical approach maintained the integrity and stability of the posterior vertebral column and decreased recovery time, achieving excellent clinical efficacy (4-9).

As microsurgery advances, the tubular retractor system has been used in microscopic discectomy for prolapsed intervertebral disc and decompression for spinal stenosis (33-36). In contrast with traditional open surgery, surgery with a tubular retractor system significantly decreases the durations of postoperative bed rest and hospital stays. This approach was characterized by minimal trauma, less hemorrhage and more rapid healing $(11,37,38)$. The Department of Neurosurgery at Union Hospital applied this tubular retractor system in the treatment of lumbar disc herniations and spinal stenosis between 2008 and 2010 with high efficacy (39). Considering the feasibility of the hemilaminectomy approach for surgical removal of the intraspinal extramedullary tumors as well as the experience gained in the practice of the tubular retractor system, the paraspinal keyhole approach was adopted in the present study in microsurgery for eligible patients with intraspinal extramedullary tumors. The present retrospective study selected patients with postoperative pathological reports indicating diagnoses of intraspinal extramedullary schwannomas.

There are several considerations that should be made when considering the application of this combined surgical technique for treatment of intraspinal schwannomas. First, patients should be placed in a prone position with their abdomens vacant. This position decreases the spinal vascular pressure and cerebrospinal fluid, decreases intraoperative hemorrhaging, facilitates surgical manipulations and avoids intracranial hypotonic headaches caused by loss of cerebrospinal fluid. Secondly, the optimal diameter of the pathway should be based on the location and size of the tumor. A minimal possible pathway ensures surgical manipulations and adequate depth-of-field of the microscopic images to decrease muscle and nerve injury.

The present study revealed that the cannula should be inserted alongside the Kirschner wire following angulation of the wire by the C-arm X-ray machine. Laterally pulling should be avoided in blunt dissection to decrease tissue damage. Movement should be avoided once the pathway is fixated. This may decrease muscle damage commonly observed when traditional surgical retractors cause excessive traction. In contrast with dilators, this method also decreases unnecessary nerve damage or nerve root irritation caused by movement.

Parts of the tumors were small and located unilaterally inside the spine and outside the spinal dura mater. Following wearing down part of the lamina using a micro drill, tumor resection was completed. For larger tumors, the incision area of the spinal dura mater exposed the upper and lower poles of the tumor. Full exposure decreased unnecessary traction and nerve root irritation during the tumor resection. Dural tenting sutures should be applied carefully to enable microscopic observation of the surgical field and to achieve epidural hemostasis.

During the initial surgical process, it was easy to separate the tumor and spinal cord along the thickened arachnoid caused by adhesions between the two tissues. As the rate of the complete tumor resection was high, initial complete resection was desirable. If a secondary surgical procedure was required for a large tumor or recurrent tumor (including the presence of a tumor closely adhering to the spinal cord), suction and electrocoagulation were utilized for careful 'peeling'. If the adhesions were not able to be fully 'peeled off', intratumoral resection may be followed by 'peeling off' the tumor capsule. Adhesions may be carefully separated between the tumor and the spinal cord or the arachnoid and nerve roots. Intraoperative manipulation should be gentle and careful. Ineffective manipulation should be minimized. Traction of the spinal cord should 
be performed gently so that irritation of the spinal cord and nerve root may be avoided. The peritumoral tortuous vessels should be protected, and the impact of resection and electrocautery on the function and blood supply of the spine should be minimized.

The dural sac should be carefully sutured following tumor resection to close the incision and decrease the incidence of cerebrospinal fluid leakage. The use of an artificial dural patch for the dural repair following resection of the epidural tumor close to the nerve roots is recommended. Application of fibrin glue to the dural repair site prevented cerebrospinal fluid from leaking, minimized the adhesions between dura mater and the surrounding tissues, and decreased the difficulty of repeat surgeries.

Minimally invasive surgery should not be deliberately performed for larger tumors which did not meet the inclusion criteria of the present study. Instead, such tumors should be treated with hemilaminectomy or posterior open laminectomy on the basis of the characteristics of the tumor for complete resection.

The mean operative time of the 56 patients in the present study was $96.21 \mathrm{~min}$. The mean intraoperative hemorrhage was $38.54 \mathrm{ml}$. After 3 days of bed rest, patients successfully ambulated with a waist guard. In the 1-month follow-up, 27 patients recovered their original physical activity and worked normally. In the last follow-up, MRI images of patients did not reveal residual tumors. The three-dimensional reconstruction of CT scans indicated complete vertebral structure without spondylolisthesis or spinal deformity.

Although the present study demonstrated satisfactory efficacy in treating lumbar intraspinal extramedullary schwannomas with the method described, further clinical evidence with a larger sample size and longer follow-up periods is required to further support the advantages of this approach. The learning curve in microneurosurgery is relatively steep (35), therefore surgical skill and experience are important factors in determining a successful outcome. The results of the present study indicate that the microsurgical paraspinal keyhole approach for lumbar intraspinal extramedullary schwannomas combined with the tubular retractor system, is feasible. Further evidence is required to exclude the biases caused by inconsistent micromanipulation of surgeons and individual differences between the patients.

In conclusion, the results of the present study suggest that the microsurgical paraspinal keyhole approach with a tubular retractor system is suitable for lumbar intraspinal extramedullary schwannomas if the surgical indications and limitations are fully understood. It is associated with several advantages including decreased hemorrhaging, decreased hospital stays and an improved postoperative maintenance of spinal stability.

\section{References}

1. Gottfried ON, Binning MJ and Schmidt MH: Surgical approaches to spinal schwannomas. Contemporary Neurosurgery 27: 1-9, 2005.

2. McCormick PC: Surgical management of dumbbell tumors of the cervical spine. Neurosurgery 38: 294-300, 1996.

3. Samii M, Babu RP, Tatagiba M and Sepehrnia A: Surgical treatment of jugular foramen schwannomas. J Neurosurg 82: 924-932, 1995 .

4. Chiou SM, Eggert HR, Laborde G and Seeger W: Microsurgical unilateral approaches for spinal tumour surgery: Eight years experience in 256 primary operated patients. Acta Neurochir (Wien) 100: 127-133, 1989.
5. Naganawa T, Miyamoto K, Hosoe H, Suzuki N and Shimizu K: Hemilaminectomy for removal of extramedullary or extradural spinal cord tumors: Medium to long-term clinical outcomes. Yonsei Med J 52: 121-129, 2011.

6. Sario-glu AC, Hanci M, Bozkuş H, Kaynar MY and Kafadar A: Unilateral hemilaminectomy for the removal of the spinal space-occupying lesions. Minim Invasive Neurosurg 40: 74-77, 1997.

7. Chiou SM, Eggert H, Laborde G and Seeger W: Microsurgical unilateral approaches for spinal tumour surgery: Eight years' experience in 256 primary operated patients. Acta Neurochir (Wien) 100: 127-133, 1989.

8. Eggert H, Scheremet R, Seeger W and Gaitzsch J: Unilateral microsurgical approaches to extramedullary spinal tumours. Operative technique and results. Acta Neurochir (Wien) 67: 245-253, 1983.

9. Sridhar K, Ramamurthi R, Vasudevan MC and Ramamurthi B Limited unilateral approach for extramedullary spinal tumours. Br J Neurosurg 12: 430-433, 1998.

10. Bertalanffy H, Mitani S, Otani M, Ichikizaki K and Toya S: Usefulness of hemilaminectomy for microsurgical management of intraspinal lesions. Keio J Med 41: 76-79, 1992.

11. Lee GW, Jang SJ, Shin SM, Jang JH and Kim JD: Clinical and radiological outcomes following microscopic decompression utilizing tubular retractor or conventional microscopic decompression in lumbar spinal stenosis with a minimum of 10-year follow-up. Eur J Orthop Surg Traumatol 24 (Suppl 1): S145-S151, 2014.

12. Chotigavanichaya C, Korwutthikulrangsri E, Suratkarndawadee S, Ruangchainikom M, Watthanaapisith T, Tanapipatsiri S and Chotivichit A: Minimally invasive lumbar disectomy with the tubular retractor system: 4-7 years follow-up. J Med Assoc Thai 95 (Suppl 9): S82-S86, 2012.

13. Kunert P, Kowalczyk P and Marchel A: Minimally invasive microscopically assisted lumbar discectomy using the METRx X-Tube system. Neurol Neurochir Pol 44: 554-559, 2010.

14. Lee CK, Park JY and Zhang HY: Minimally invasive transforaminal lumbar interbody fusion using a single interbody cage and a tubular retraction system: Technical tips, and perioperative, radiologic and clinical outcomes. J Korean Neurosurg Soc 48: 219-224, 2010.

15. Schwartz TH and McCormick PC: Spinal cord tumors in adults. In: Youmans Neurological Surgery. Winn HR (ed). Vol 4. 5th edition. pp4817-4834. WB Saunders Philadelphia, PA, USA, 2004.

16. Devereaux MW: Neck and low back pain. Med Clin North Am 87: 643-662, 2003.

17. Wu G, Ghimire P, Zhu L, Baral A and Su J: Magnetic resonance imaging characteristics of primary intraspinal peripheral primitive neuroectodermal tumour. Can Assoc Radiol J 64: 240-245, 2013.

18. Teksam M, Casey SO, Michel E, Benson M and Truwit CL: Intraspinal epidermoid cyst: Diffusion-weighted MRI. Neuroradiology 43: 572-574, 2001.

19. Kukreja K, Manzano G, Ragheb J and Medina LS: Differentiation between pediatric spinal arachnoid and epidermoid-dermoid cysts: Is diffusion-weighted MRI useful? Pediatr Radiol 37: 556-560, 2007.

20. Tang L, Cianfoni A and Imbesi SG: Diffusion-weighted imaging distinguishes recurrent epidermoid neoplasm from postoperative arachnoid cyst in the lumbosacral spine. J Comput Assist Tomogr 30: 507-509, 2006.

21. McCormick PC, Post KD and Stein BM: Intradural extramedullary tumors in adults. Neurosurg Clin N Am 1: 591-608, 1990.

22. Dickman C, Fehlings M and Gokaslan ZL: Spinal Cord and Spinal Column Tumors: Principles and Practice. Thieme, New York, NY, pp712, 2006.

23. Hussein AA, El-Karef E and Hafez M: Reconstructive surgery in spinal tumours. Eur J Surg Oncol 272: 196-199, 2001.

24. Seppälä MT, Haltia MJ, Sankila RJ, Jääskeläinen JE and Heiskanen O: Long-term outcome after removal of spinal schwannoma: A clinicopathological study of 187 cases. J Neurosurg 83: 621-626, 1995.

25. Sridhar K, Ramamurthi R, Vasudevan MC and Ramamurthi B: Giant invasive spinal schwannomas: Definition and surgical management. J Neurosurg 94 (Suppl 2): S210-S215, 2001.

26. Zander T, Rohlmann A, Klöckner C and Bergmann G: Influence of graded facetectomy and laminectomy on spinal biomechanics. Eur Spine J 12: 427-434, 2003.

27. Wigfield CC, Skrzypiec D, Jackowski A and Adams MA: Internal stress distribution in cervical intervertebral discs: The influence of an artificial cervical joint and simulated anterior interbody fusion. J Spinal Disord Tech 16: 441-449, 2003. 
28. Sköld C, Tropp H and Berg S: Five-year follow-up of total disc replacement compared to fusion: A randomized controlled trial. Eur Spine J 22: 2288-2295, 2013.

29. Wei J, Song Y, Sun L and Lv C: Comparison of artificial total disc replacement versus fusion for lumbar degenerative disc disease: A meta-analysis of randomized controlled trials. Int Orthop 37: 1315-1325, 2013

30. Chang UK, Kim DH, Lee MC, Willenberg R, Kim SH and Lim J: Changes in adjacent-level disc pressure and facet joint force after cervical arthroplasty compared with cervical discectomy and fusion. J Neurosurg Spine 7: 33-39, 2007.

31. Park AE and Heller JG: Cervical laminoplasty: Use of a nove titanium plate to maintain canal expansion-surgical technique. J Spinal Disord Tech 17: 265-271, 2004.

32. Acioly MA, Carvalho CH, Koerbel A, Heckl S, Tatagiba M and Gharabaghi A: The role of the trigeminocardiac reflex in postoperative hearing function in non-vestibular schwannoma cerebellopontine angle tumors. J Clin Neurosci 18: 237-240, 2011.

33. Palmer S: Use of a tubular retractor system in microscopic lumbar discectomy: 1 year prospective results in 135 patients. Neurosurg Focus 13: E5, 2002.
34. Parikh K, Tomasino A, Knopman J, Boockvar J and Härtl R: Operative results and learning curve: Microscope-assisted tubular microsurgery for 1- and 2-level discectomies and laminectomies. Neurosurg Focus 25: E14, 2008.

35. Ranjan A and Lath R: Microendoscopic discectomy for prolapsed lumbar intervertebral disc. Neurol India 54: 190-194, 2006.

36. Yoshimoto M, Takebayashi T, Kawaguchi S, Tsuda H, Ida K, Wada T, Suzuki D and Yamashita T: Minimally invasive technique for decompression of lumbar foraminal stenosis using a spinal microendoscope: Technical note. Minim Invasive Neurosurg 54: 142-146, 2011.

37. Foley KT, Smith MM and Rampersaud YR: Microendoscopic approach to far-lateral lumbar disc herniation. Neurosurg Focus 7: e5, 1999.

38. Fessler RG and Khoo LT: Minimally invasive cervical microendoscopic foraminotomy: An initial clinical experience. Neurosurgery 51 (Suppl 5): S37-S45, 2002.

39. Chunmei Chen, Fenggang Cai, Weiqiang Zhang, Rui Wang, Yan Chen, Feng Li, et al: The comparison between paraspinal sleeve approach with posterior midline approach microsurgical treatment of lumbar disc herniation. Chin J Neurosurg 30: 677-681, 2014 (In Chinese). 